\title{
EXPERIMENTAL INVESTIGATION ON ELASTO-PLASTIC DEFORMATION OF IS:2062 GRADE C STEEL IN THE POST YIELD REGION
}

\author{
D Venkatesh, Balasubramanya H S, Naveen Kumar B K \\ Ramaiah Institute of Technology, Bangalore, India \\ S V Prakash \\ Sri Krishna Institute of Technology, Bangalore, India \\ Anand Rajeev and Sujitha R \\ Ramaiah Institute of Technology, Bangalore, India
}

\begin{abstract}
Rapid growth and tough competition in material processing industries such as automobile and aerospace demands precise components to be processed in short time. The processing steps involved in fabrication of intricate shaped final product involves various intermediate steps such as design, selection of material and manufacturing process etc. In these steps, a thorough understanding of material plastic behavior plays a vital role. Currently there is no acceptable model is available for a range of applications that gives solution to practical and theoretical issues. Most of the theories are either related to material or can be applied only to specific processes. These theories overgeneralized the physical real world situation using assumptions and empirical relations. Deformation involves changes the shape and size. Composites and other material exhibits some sort of inelasticity even under very low stresses. Constitutive relation can't treat the elastic and plastic deformations separately. Classical yield criteria fail to correlate the elastic plastic behavior for material deformation in 3-D.

In the present investigation new generalized relation has been developed by employing the concept of activation energy to represent the material behaviour from elastic range to failure. This relation involves eight material parameters which includes two classical material constants like bulk modulus and shear modulus, two material viscosity terms and four in the form rule which relates elastic strain rate to plastic strain rate. All these material parameters can be obtained by conducting simple experiments.

Experiments are carried out to find material parameter values. In the present work, experiments on the commercial grade mild steel and aluminum are discussed.
\end{abstract}

Keywords: classical theory, deviatoric strain, activation energy. 
Cite this Article: D Venkatesh, Balasubramanya H S, Naveen Kumar B K, S V Prakash, Anand Rajeev and Sujitha R, Experimental investigation on elasto-plastic deformation of IS:2062 Grade C steel in the post yield region, International Journal of Mechanical Engineering and Technology 11(5), 2020, pp. 54-60.

https://iaeme.com/Home/issue/IJMET?Volume $=11 \&$ Issue $=5$

\section{INTRODUCTION}

Today, technology is one of the bastions of our modern lifestyle and the basis for our prosperity, in which metal forming technology plays a central role. There is a large number of products that everyone use daily and that have been produced entirely or partially by metal forming. Vehicles, furniture, electrical appliances are only few products of this endless list. Once a deformed sheet-metal part is removed from the tooling, elastic recovery will occur, especially where bending, bending-unbending, and reverse bending are performed. This phenomenon is called spring back. In the automobile industry the car body structure is made of a large number of sheet metal parts. These parts, usually with rather complex geometries, make use of different materials varying from mild steels to very high strength steels or aluminium depending on the product requirements and the allowed costs. Sheet metal forming as blanking, punching, bending and stamping technologies is nowadays a key issue among the industrial manufacturing processes in the automotive, aeronautic, kitchen devices, packaging industries and others. It consists of deformation processes in which a flat metal blank is shaped by tools or dies under the action of biaxial stretching, deep drawing and bending deformation modes to produce the desired product or piece without ruptures or defects. In metal forming, a piece of material is plastically deformed between tools to obtain the desired shape. A commonly used sheet metal forming process is the stretch forming process, which is frequently used in the automotive industry (outer panels, inner panels, stiffeners etc.), the packaging industry and in the household appliances sector to manufacture complicated shapes and curvatures.

\section{MATERIAL SELECTION AND EXPERIMENTAL METHODS}

In the present work Commercial grade mild steel (IS2062) is selected for the investigation. Standard sized specimens are prepared confirming to ASTM 8/E 8M-08 standard, are tested under condition of uni-axial tension on a hydraulic controlled tensile testing machine having $160 \mathrm{kN}$ capacity. Experiments are carried out for five different strain rates varying from 1E-04 to $1.7 \mathrm{E}-03$ per second. Five samples are chosen for each strain rate for the repeatability of material parameters during deformations. Since specimen is not axi-symmetric, strains are measured in the two lateral directions also. Load is applied in axial direction and strain is measured in three directions, i.e. axial, lateral and across the thickness of the specimen. Extensometers are used instead of standard strain gauges to avoid any inaccuracies in the data occurring in the post yield region due to failure of strain gauges. Three extensometers of $0-12.5$ $\mathrm{mm}$ range are fixed at the centre of the specimen to record the strains in all the three directions. Strain, load and dimensions are measured within accuracy of $\pm 0.001, \pm 0.001 \mathrm{kN}$ and $\pm 0.05 \mathrm{~mm}$ respectively. All measurements are carried out till the failure of the specimen. Elastic constants, bulk modulus $(\mathrm{K})$ and shear modulus $(\mathrm{G})$ are obtained at very small strains and assumed to remain constant during deformation. Strain rates are used to find material viscosity parameters, bulk viscosity $\left(\mu^{\prime}\right)$ and material shear viscosity $(\mu)$. Using elastic constants, elastic strains and plastic strains are separated out. Corresponding components of elastic strain rate and plastic strain rates are used to find remaining four constants associated with volumetric and deviatoric strain rates. The material behaviour in the post yield region with new understanding of the material model is presented here for uni-axial loading case. Based on the experimental observations and the results obtained it is found that the both the elastic moduli and the 
viscosities remain constant throughout the large deformation. The region around the yield point is modeled to represent the plastic strain rates. Both volumetric and deviatoric elastic and plastic strains contributions and their variations are observed and the corresponding stresses are computed. The effect of strain rate on plastic stress is investigated. The machine set up is as shown in Fig. 1 and Fig. 2.

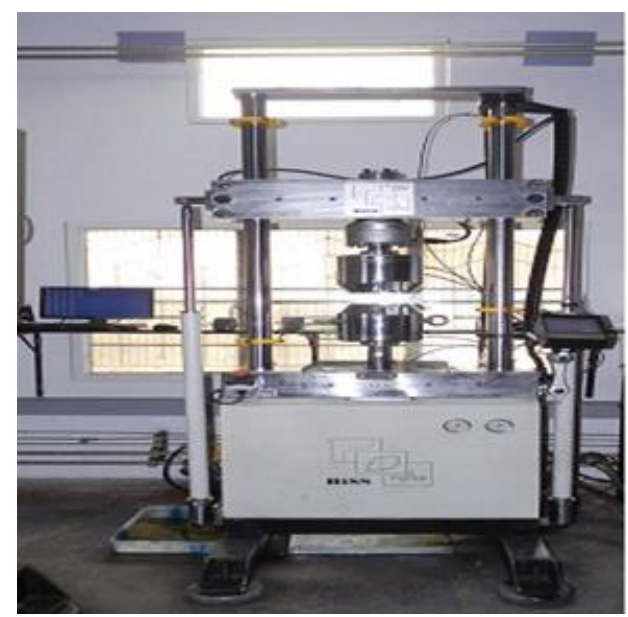

Figure 1 Experimental Setup

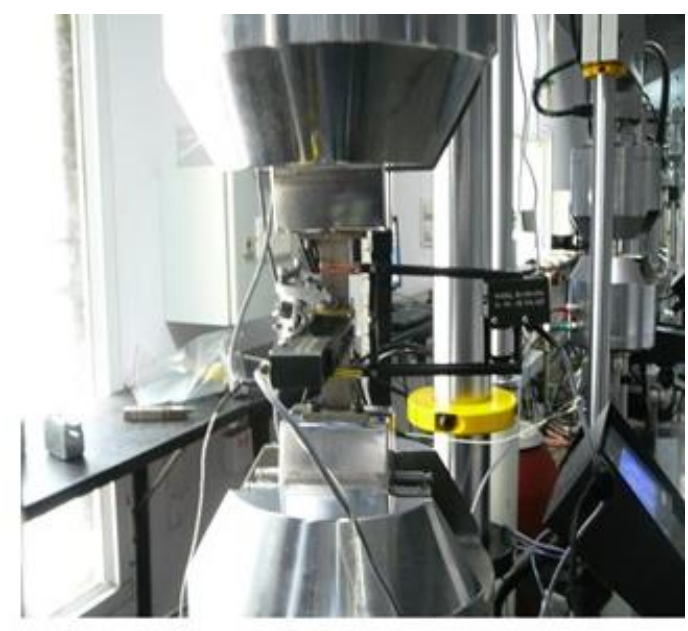

Figure 2 Experimental Setup with specimen

Test samples are made as per ASTM 8/E 8M-08. Sample dimensions are shown in Fig. 3. All the dimensions of the sample are ensured as per ASTM 8/E 8M-08 and samples are numbered. Gage length marking is done on each sample by scribing lightly.

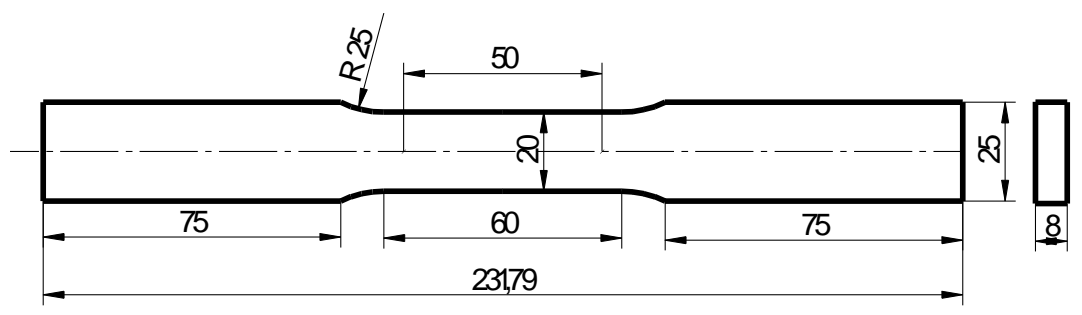

Figure 3 Mild steel specimen Dimensions as per ASTM 8/E 8M-08.

Basic data obtained from the experiments are, applied axial load, axial and lateral strains as functions of time and at different strain rates. Chemical and physical properties of the materials are chosen such that they are closely related to those used in the automotive industry for stretch forming. All experiments are carried out at room temperature with constant strain rates varying from $100 \mu$ Strains to $1700 \mu$ Strains. The experimental data are checked for repeatability.

\subsection{Field equations}

The new field equation consists of resistance from strain and dissipative part due to strain rate. The stress relation for a material during deformation can be expressed as

$$
\begin{gathered}
\sigma=\sigma_{\mathrm{e}}+\sigma_{\mathrm{p}} \\
\sigma_{e}=K \Theta_{e} I+2 G\left(\varepsilon_{e}-\frac{\Theta_{e}}{3} I\right) \\
\sigma_{p}=\mu^{\prime} \dot{\Theta}_{p} I+2 \mu\left(\dot{\varepsilon}_{p}-\frac{\dot{\Theta}_{p}}{3} I\right)
\end{gathered}
$$

Considering the contribution from the strain and strain rate parts, the stress is expressed as 


$$
\sigma=K\left(\Theta_{e}\right) I+2 G\left(\varepsilon_{e}-\frac{\Theta_{e}}{3} I\right)+\mu^{\prime} \frac{\partial}{\partial t}\left(\Theta_{p}\right) I+2 \mu \frac{\partial}{\partial t}\left(\varepsilon_{p}-\frac{\dot{\Theta}_{p}}{3} I\right)
$$

where $\Theta_{e}$ and $\Theta_{p}$ are the elastic and plastic parts of the volumetric strain respectively. Elastic moduli, $\mathrm{K}$ and $\mathrm{G}$ are constants associated with the elastic volumetric and the elastic deviatoric strains respectively. The other two material parameters, material viscosities $\mu$ and $\mu^{\prime}$, are also constants and associated with the plastic strain rate part. The plastic parts of the volumetric and deviatoric strains have appeared only as strain rates. These plastic parts represent the energy loss in the form of dissipation during deformation.

\section{EXPERIMENTAL DATA ANALYSIS}

From experiments, the basic data available are, applied load, axial and lateral strains as functions of time and at different strain rates. During uniaxial tension test, the applied stress in the lateral direction is zero. i.e., $\sigma_{2}=\sigma_{3}=0$.

$$
\varepsilon^{\prime} 1 \mathrm{p}=(\mathrm{d} / \mathrm{dt}) \varepsilon 1 \mathrm{p} ; \varepsilon^{\prime} 2 \mathrm{p}=(\mathrm{d} / \mathrm{dt}) \varepsilon 2 \mathrm{p} ; \varepsilon^{\prime} 3 \mathrm{p}=(\mathrm{d} / \mathrm{dt}) \varepsilon 3 \mathrm{p} .
$$

There are three equations and six unknown quantities $\varepsilon_{1 \mathrm{e}}, \varepsilon_{2 \mathrm{e}}, \varepsilon_{3 \mathrm{e}}, \varepsilon_{1 \mathrm{p}}, \varepsilon_{2 \mathrm{p}}$, $\varepsilon_{3 \mathrm{p}}$ in the form of strain field provided the material constants, $\mathrm{K}, \mathrm{G}, \mu^{\prime}, \mu$ are known to solve the system. the plastic strains can be expressed in terms of total strain and elastic strain. Plastic strain component and expressed as

$$
\varepsilon 1 \mathrm{p}=\varepsilon 1-\varepsilon 1 \mathrm{e} ; \varepsilon 2 \mathrm{p}=\varepsilon 2-\varepsilon 2 \mathrm{e} ; \varepsilon 3 \mathrm{p}=\varepsilon 3-\varepsilon 3 \mathrm{e}
$$

\section{MEASUREMENT OF MATERIAL PARAMETERS}

The values of material constants $\mathrm{K}, \mathrm{G}, \mu^{\prime}, \mu$ can be found out using experiments at small strains and strain rates since material constants are unaffected by material non-linearities, which become significant at higher strain and strain rates. The property values for mild steel is provided in the Table 1

Table 1 Material properties of Mild steel

\begin{tabular}{|c|c|}
\hline \multirow{2}{*}{ Parameters } & Values \\
\cline { 2 - 2 } & Mild steel \\
\hline Young's modulus, E & $209.42 \mathrm{GPa}$ \\
\hline Bulk modulus, $\mathrm{K}$ & $193.52 \mathrm{GPa}$ \\
\hline Rigidity modulus, G & $79.17 \mathrm{GPa}$ \\
\hline Poisson's ratio & 0.32 \\
\hline
\end{tabular}

The system is solved for elastic strains using the known values of applied stress, total strains in axial and lateral directions and the rate at which these experiments are carried out. Now by knowing the elastic strains, plastic strain and plastic strain rates are calculated.

\section{RESULTS AND DATA ANALYSIS}

The experimental data for commercially available mild steel specimen (IS:2062 Grade C) at different strain rates are shown in Fig. 4. All analysis are carried out on these set of experimental data. The contribution from the axial elastic and plastic strain on total axial strain is shown in Fig. 5. While, Fig. 6 shows for the lateral strains along width. In the elastic or earlier part of the deformation region, most of the contribution for total strain comes from elastic strain. In this region, plastic strains are very small or nearly zero. During the later part of deformation, the contribution from the elastic strain is very less and total contribution remains constant. After yielding, plastic strain contributes more to the total strain. Major part of the total strain consists 
of only of plastic strain. Similar behavior is observed in the case of lateral strains also. The major contribution for stress comes from the elastic strain part.

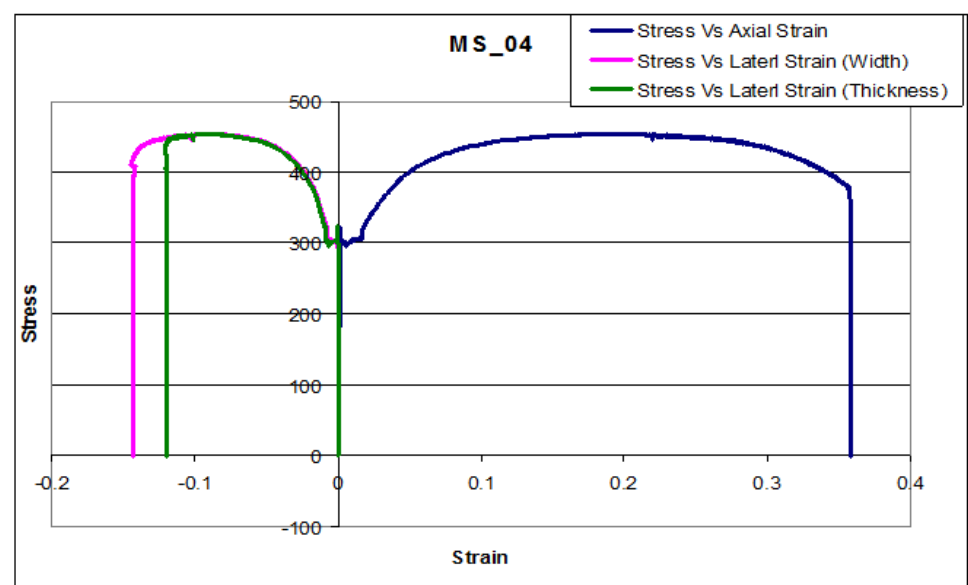

Figure 4 Stress v/s strain diagram for mild steel specimen

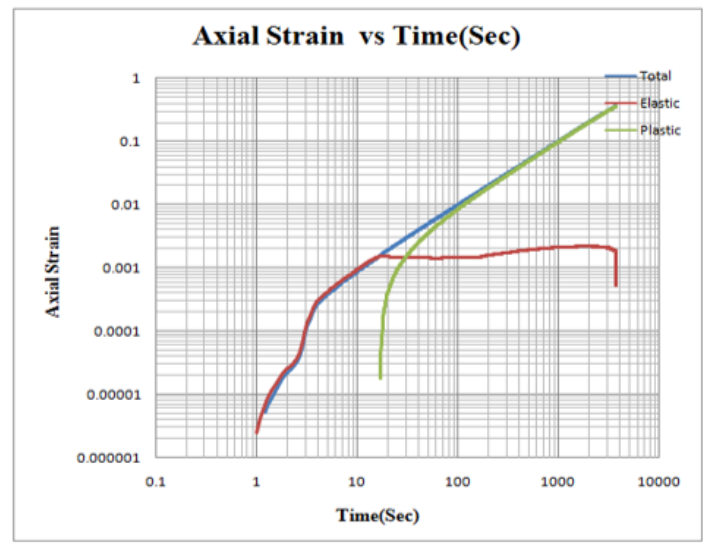

Figure 5 Strain in axial direction v/s time for MS specimen

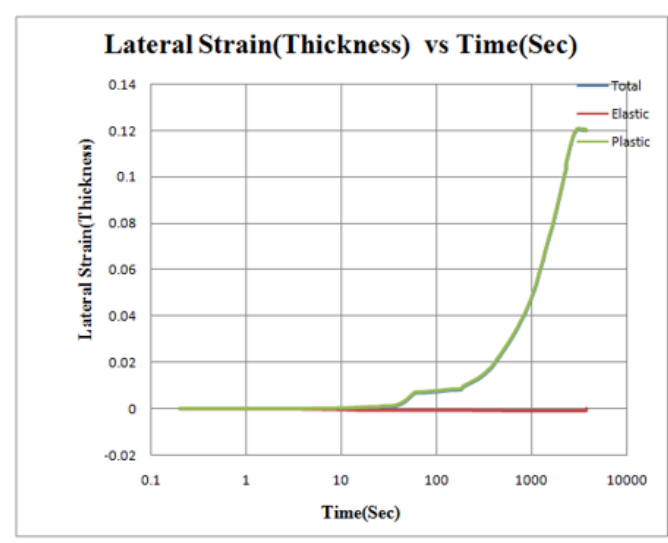

Figure 6 Lateral strain v/s time for MS specimen

Fig. 7, Fig. 8, Fig. 9, Fig. 10 shows the behavior of volumetric strain and deviatoric strain with deformation for mild steel. These figures show the contribution from the elastic and plastic parts. Elastic volumetric strain increases with deformation in the beginning and after yielding, there is a very little change which shows that material is compressible in the elastic region. These observations suggest slipping between grain boundaries occurring inside the material without contributing to any kind of elastic strain. During large deformation, major contribution comes from plastic deviatoric strain which is almost 4-5 timesmore than plastic volumetric strain. The contribution from the elastic deviatoric strain during large deformation is almost constant. Their presence indicates the elastic unloading at any point in plastic range.In the case of mild steel (Fig. 6.11 and 6.12), the volumetric and deviatoric plastic strains are almost zero during earlier part of deformation. Around yielding, there is a sharp increase in both volumetric and deviatoric plastic strain rates. After yielding, a drop is observed in these plastic strain rates. Like aluminium alloy, mild steel also shows that the deviatoric plastic strain rate is almost one order more than the corresponding volumetric plastic strain rate. 


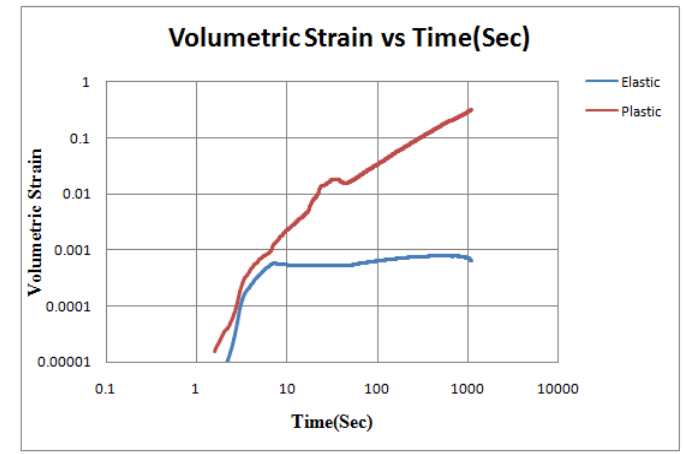

Figure 7 Behavior of volumetric strain vs Time

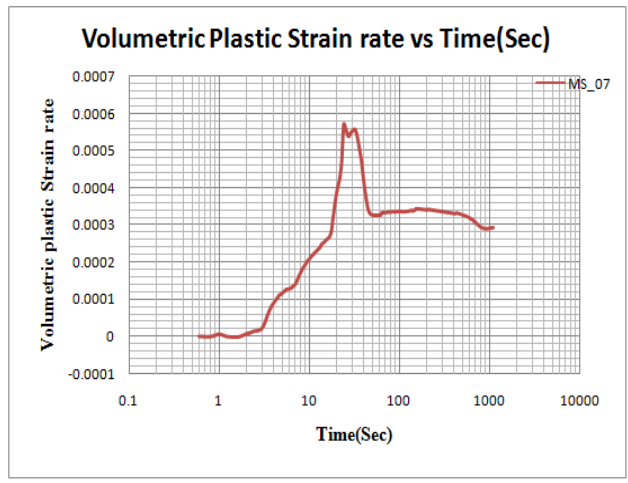

Figure 9 Volumetric plastic strain rate vs time

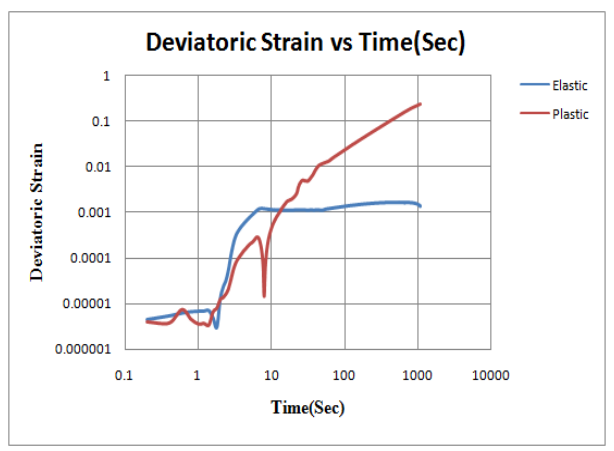

Figure 8 Behavior of Deviatoric strain vs Time

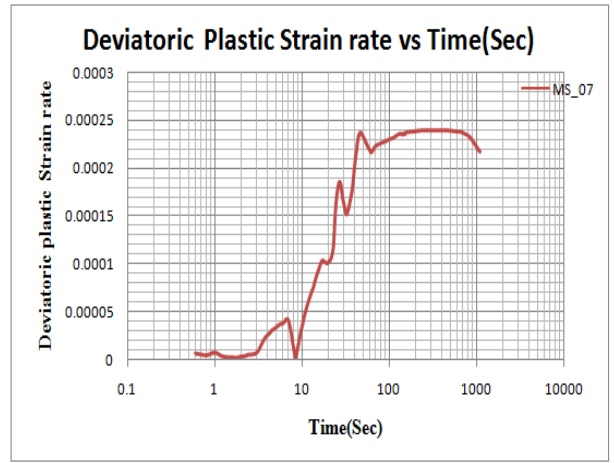

Figure 10 Deviatoric plastic strain rate vs time

Plastic rate constants:-The other four material constants, two activation points $(\Theta y, J 2 y)$ and two plastic rate constants $(\phi, \psi)$, which relate the elastic strain rate with plastic strain rate are obtained using experimental data. Fig. 10 and 11 shows the behavior of volumetric plastic strain rate to elastic strain rate ratio for mild steel. Fig. 11 shows the behavior of volumetric plastic strain rate to volumetric elastic strain rate ratio with respect to volumetric elastic strain, while Fig. 12 shows the behavior for deviatoric plastic strain rate to deviatoric elastic strain rate ratio with respect to second invariant of deviatoric elastic strain tensor. In both these graphs, after some initial deformation, the slope of strain rate ratio for different strain rates remains the same. the values of this slope is considered as $\phi$ and $\psi$. The farthest point at which the slope meets the $\mathrm{x}$-axis is considered as activation points, Өy and $\mathrm{J} 2 \mathrm{y}$ as shown in graph. The calculated values of these eight material constants for mild steel specimen are tabulated in Thesis.

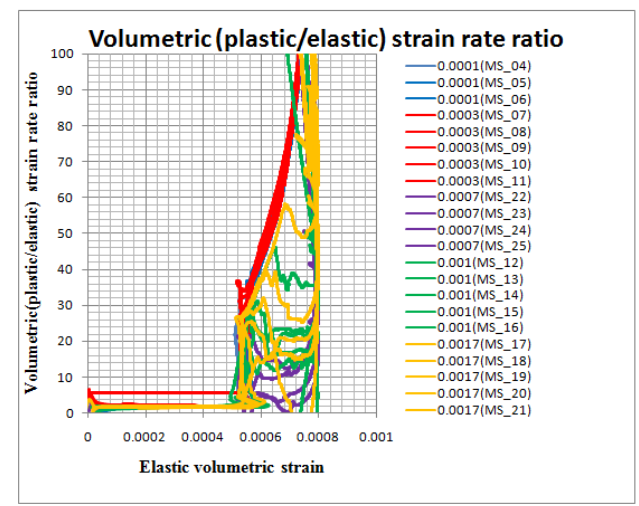

Figure 11 Volumetric plastic strain rate ratio vs

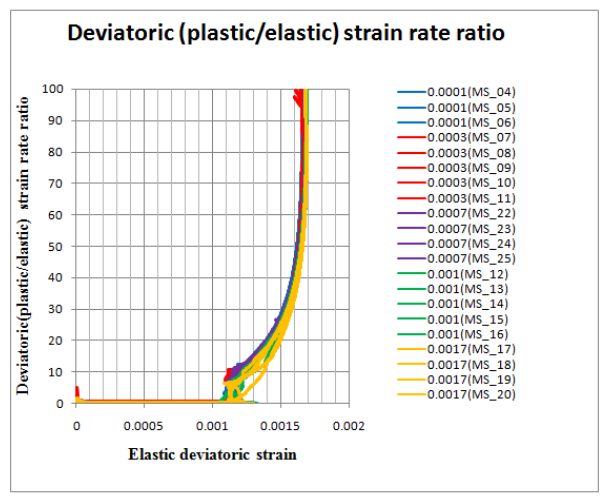

Figure 12 Deviatoric plastic strain rate ratio $\varepsilon_{\mathrm{v}}$ VS $\varepsilon_{\mathrm{d}}$ 
Experimental investigation on elasto-plastic deformation of IS:2062 Grade C steel in the post yield region

\section{CONCLUSION}

In conclusion, the experimental behavior reveals the change in plastic volume along with elastic volume change. The use of concept like activation energy to separating the elastic strain and plastic strain, plastic flow, etc. have a better physical insight than the classical yield theories. the major contribution during plastic flow comes from the plastic strain and the plastic stress hardly contributes anything to the total stress. The major part of the work done during deformation is due to the elastic stress than due to plastic stress.

\section{REFERENCES}

[1] Gotoh, M., Yamashita, M., 2003. An aspect of plasticity with compressibility. International Journal of Plasticity 19, 383.

[2] Hill, R., 1950. Mathematical Theory of Plasticity. Oxford University Press, London.

[3] Jain, P., 2006. Measurements and simulation of elasto-plastic deformation. Msc thesis, Dept of Mechanical Engineering, Indian Institute of Science, Bangalore.

[4] Karthikeyan, P., 2004. A new approach to process modeling and simulation of metal forming. M.Sc. thesis, Dept of Mechanical Engineering, Indian Institute of Science, Bangalore.

[5] Jiang, Y., Sehitoglu, H., 1994a. Multi-axial cyclic ratchetting under multiple step loading. International Journal of Plasticity 10, 849.

[6] Jiang, Y., Sehitoglu, H., 1994b. Cyclic ratchetting of 1070 steel under multiaxial stress states. International Journal of Plasticity 10, 579.

[7] Khan, A.S., Huang, S., 1995. Continuum Theory of Plasticity. John Wiley and sons, New York.

[8] Rao, A.R., Shrinivasa, U., 2002. Towards simulation of elasto-plastic deformation: an investigation. Sadhana 27, 251. 